\title{
Energy Reconstruction Techniques in TileCal under High Pile-up Conditions
}

\author{
G. I. Gonçalves ${ }^{1,2}[0000-0002-6807-3172]$ \\ on behalf of the ATLAS Tile Calorimeter Group \\ ${ }^{1}$ Federal University of Rio de Janeiro, RJ, Brazil \\ ${ }^{2}$ Computational Modeling Graduate Program, Rio de Janeiro State University, \\ Brazil
}

\begin{abstract}
Particle colliders are machines built to probe fundamental questions in physics. The properties of the produced particles are measured by complex experiments which use a wide variety of devices, such as the calorimeter system. In the ATLAS experiment at LHC, the Tile Calorimeter (TileCal) comprises about 10,000 readout channels that amplify, shape, and sample each signal every 25 ns. Since LHC collisions occur every $25 \mathrm{~ns}$, and due to the increase of the luminosity level, signals from adjacent collisions may be read out within the same TileCal readout window, deforming the expected signal, and degrading the energy estimation efficiency. Therefore, this work compares the performance of the currently available methods for TileCal energy estimation using LHC collision data. Different pile-up conditions are considered. The results show that the performance in terms of the uncertainty of the energy estimation can be improved up to $35 \%$ in high-occupancy readout channels.
\end{abstract}

Keywords: Optimal filter · Signal estimation · Wiener filtering · Pile-up - High-energy calorimetry.

\section{Introduction}

The electronics for high-energy physics experiments deal with enormous technological challenges as a large amount of data needs to be processed within a short time. The calorimeter systems of such complex experiments play an important role as they are used to measure the energy of incident particles. The information provided by the calorimeter systems is used for event reconstruction and particle identification [1].

Typically, a calorimeter is segmented into readout cells (tens of thousands in modern calorimeters), providing spatial resolution to the detector. The readout signals are processed by a pulse-shaping electronic circuit, which gives the pulse of a well-defined shape with an amplitude proportional to the particle energy [2]. Thus, the problem of energy estimation can be stated as determining the amplitude of the pulse produced by the calorimeter readout channel. Energy is the most important information in high-energy calorimetry systems, being a fundamental property for the reconstruction of events and validation of models for physical phenomena. 
The commonly employed mathematical methods for energy estimation formulate the problem as estimating the amplitude of a pulse immersed in additive noise, where the identification of channels with relevant information is performed through an energy threshold at the output of the estimator. Usually, when modeling the problem, these approaches consider a fixed pulse shape and additive Gaussian noise [3,4].

However, modern colliders, such as the LHC [5], operate with high event rates and high luminosity levels. The luminosity is defined as a proportional factor between the number of events per second and the interaction cross section, having the unit of $\mathrm{cm}^{2} \mathrm{~s}^{-1}[6]$. In such conditions, the particle density in the beam cross-section is increased so that many interactions could occur at each collision point, generating more signals in the detectors, such as the calorimeters [7]. As a result, the energy estimation problem becomes more complex because of that can be observed where two or more pulses are acquired within the same readout window. To mitigate the, new methods based on the deconvolution of superimposed signals have been proposed [8,9]. Additionally, an approach based on Wiener filtering has also been tested for severe conditions [10].

The ATLAS (The Toroidal LHC AparatuS) experiment [11] covers a wide spectrum of physics of interest at the LHC, and the information from its calorimeter system is important for the complex trigger system which selects only the relevant information from the collisions to be stored. Future LHC upgrades are planned to increase the collision energy and the luminosity level. The increase in luminosity raises the number of proton-proton interactions per collision, producing more data and increasing the probability of observing events of interest. However, in the calorimeter system, signals from neighboring events that occurred in different time may be read out causing the effect which degrades the performance of typical energy estimation methods. In the Tile Calorimeter (TileCal) [12] of the ATLAS, three energy estimation methods address the in different ways. Therefore, this work evaluates the performance of these methods in different conditions using collision data acquired during 2018 data-taking.

In the next section, the TileCal is briefly introduced. In Section 3 the methods used in TileCal for energy estimation are described. The results describing the performance analysis of the methods using real proton-proton collision data acquired in 2018 in ATLAS are presented in Section 4. Finally, in Section 5 conclusions are derived.

\section{The ATLAS Tile Calorimeter}

The ATLAS calorimetry system is sectioned according to the interaction nature: electromagnetic (electrons and photons) and hadronic (e.g. protons and neutrons). The Tile Calorimeter (TileCal) is the central hadronic calorimeter of ATLAS and provides accurate measurements of jet energy, and assists in the missing transverse moment calculation and muon detection.

TileCal is a sampling calorimeter that uses scintillating plastic plates (or tiles) as the active material, interspersed with steel layers as the absorbent ma- 
terial, according to the illustration of a module depicted in Fig. (1). Both sides of each tile are connected to a specific type of optical fiber, called WLS (Wavelength Shifting), composed of a photo-fluorescent material that absorbs a highfrequency photon and emits multiple low-frequency photons. Multiple tiles are grouped into cells and each cell is connected to two photo-multipliers (PMTs). TileCal is composed of four partitions (EBC, LBC, LBA, and EBA). Each partition is divided into 64 modules with 32 to 48 readout channels per module, producing approximately 10.000 readout signals per collision.

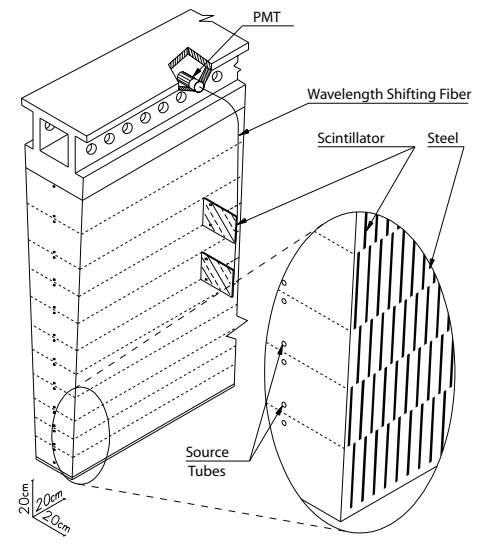

Fig. 1: Schematic diagram of a single TileCal module (extracted from [13]).

When a hadronic particle passes through the calorimeter, it generates a shower of secondary particles and loses energy by the interaction with the passive material (steel as an absorbent material) and also interacts with the active material (scintillating tiles), emitting light. This light is conducted by optical fibers and read out by PMTs, which generate an electrical pulse in response to the light signal. The pulse generated by the PMT is conditioned by a conformation circuit (shaper), which provides a pulse with a known shape and amplitude proportional to the deposited energy [14]. This analog pulse is digitized by an Analog to Digital Converter (ADC) with a sampling frequency of $40 \mathrm{MHz}$. A window with seven time samples is available to extract the parameters from the readout pulse.

The deposited energy in each cell of the calorimeter can be calculated by correctly estimating the pulse amplitude, which is an approach commonly used in modern calorimeters. Physically, only cells located along the shower development should contain energy deposits and are selected for energy reconstruction [15].

The average number of interactions per collision $\langle\mu\rangle$ is used to represent how occupied a given readout cell is at each collision. Currently, the LHC operates with $\langle\mu\rangle$ approximately equal to 40 , and this value should increase for Run 3 [16]. As a consequence of the increase in luminosity, the probability of the 
occurrence of signals within the same TileCal readout channel also increases. When signals from adjacent events are acquired within a same readout window, the effect is observed as illustrated by Fig. (2).

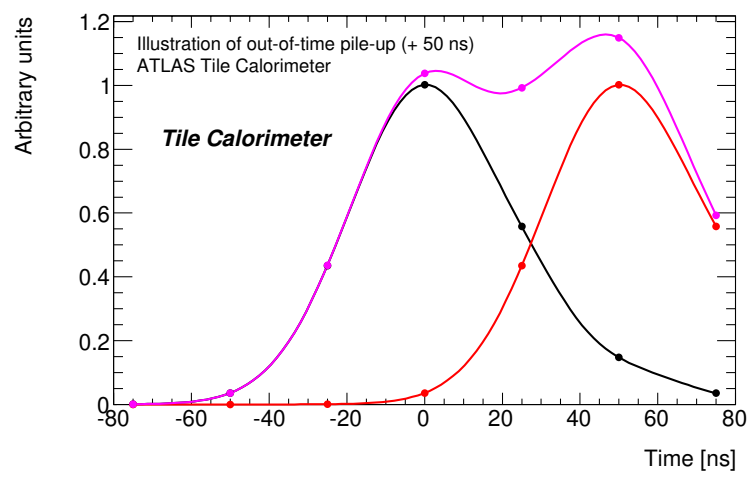

Fig. 2: An illustration of the pile-up effect. The black pulse is the signal of interest and the red one is the time-shifted signal. The resulting received pulse is in magenta (extracted from [17]).

\section{Energy estimation in TileCal}

The problem of the energy reconstruction in calorimeters is commonly addressed as a parameter estimation problem. Currently, TileCal has three methods available for energy reconstruction of signals from collisions: the Optimal Filter, the Constrained Optimal Filter (COF), and the Wiener Filter. The methods address the in different ways as described below.

\subsection{The Optimal Filter}

The Optimal Filter (OF) method aims to estimate the amplitude of an input signal, which is proportional to the energy. This estimator is designed to minimize the estimation variance using the knowledge of the pulse shape (output of the shaper circuit). The main noise source in ATLAS calorimeters readout channels is the electronics, which can be modeled by a Gaussian distribution. Under this constraint, the filter operates optimally which makes this method widely used in ATLAS $[18,19]$.

The implemented version of this estimator in TileCal is called OF2 and has been in operation since 2014, being used for online and offline energy reconstruction [3]. This method is also used in other calorimeters in ATLAS, such as the Liquid Argon Calorimeter (LAr) [18]. 
In the OF2 method, the digital sample $x[k]$ at time $k$ can be modeled by

$$
x[k]=A g[k-\tau]+n[k]+p e d,
$$

where $A$ is the pulse amplitude, $g[k]$ the reference pulse values (shaper), $n[k]$ the additive noise, $\tau$ the phase shift of the signal, and ped the pedestal or baseline, a constant parameter added to the analog signal before its digitization.

The input signal amplitude is estimated through a low computational cost process, which uses a weighted sum operation given by

$$
\hat{A}_{O F}=\sum_{k=0}^{N-1} x[k] w[k],
$$

where $w[k]$ is the filter coefficient and $x[k]$ is the sample of the received signal.

To calculate the OF2 coefficients $w[k]$, the digital signal received from TileCal can be approximated by a first-order Taylor series, given by

$$
x[k]=A g[k]-A \tau \dot{g}[k]+n[k]+p e d,
$$

where $\dot{g}[k]$ represents the derivative of the reference pulse $g$, and $k=0,1,2, \ldots, N-$ 1.

To guarantee an unbiased estimator, the absence of estimation bias, the expected value of $\hat{A}_{O F}$ is required to equal $A$. Therefore, replacing Eq. (3) in (2) and considering that the average noise is zero $(\mathbb{E}\{n[k]\}=0)$, where $\mathbb{E}$ represents the expectation operator, the expected amplitude value becomes

$$
\mathbb{E}\left\{\hat{A}_{O F}\right\}=\sum_{k=0}^{N-1}(A w[k] g[k]-A \tau w[k] \dot{g}[k]+w[k] p e d),
$$

and

$$
\mathbb{E}\left\{\hat{A}_{O F}\right\}=A .
$$

For the estimator to be independent of the pedestal and the phase, the following restrictions are established

$$
\sum_{k=0}^{N-1} w[k] g[k]=1, \sum_{k=0}^{N-1} w[k] \dot{g}[k]=0, \text { and } \sum_{k=0}^{N-1} w[k]=0 .
$$

The first restriction guarantees an unbiased estimator, while the second and third restrictions guarantee, respectively, that the estimator is immune to phase and pedestal fluctuations.

The estimator's variance is given by

$$
\begin{aligned}
\mathbb{E}\left\{\left(\hat{A}_{O F}-A\right)^{2}\right\} & =\sum_{k=0}^{N-1} \sum_{j=0}^{N-1} w[k] w[j] C[k, j] \\
& =\mathbf{w}^{T} \mathbf{C} \mathbf{w}
\end{aligned}
$$


where $\mathbf{w}$ is the weights vector of the estimator and $\mathbf{C}$ the noise covariance matrix.

To determine the weights $\mathbf{w}$, it is necessary to minimize the expression of the estimator's variance using the Lagrange multiplier method. The solution of this system results in the set of weights $w[k]$ of the OF2 estimator that currently operate online and offline in TileCal. It is worth mentioning that currently the noise covariance matrix $\mathbf{C}$ is approximated by the identity matrix, which does not take into account the effect.

\subsection{The COF Method}

Another algorithm also available for offline reconstruction in TileCal is the COF method (Constrained Optimal Filter). COF computes a linear transformation that recovers the amplitude of superimposed signals for a given readout window. Hence, the central pulse, assigned to the collision of interest, can be dissociated and reconstructed [8].

In this respect, the COF method models the energy deposition in a given calorimeter cell as a Kronecker delta function [20], which produces an output corresponding to the TileCal reference pulse. Thus, considering a set of energy depositions $a[k]$, the received signal can be modeled as

$$
x[k]=\sum_{i}(g[i] a[n-i])+n[k] .
$$

In this way, estimating the deposited energy in a given calorimeter cell implies deconvolution of the sequence $x[k]$ of the impulse response $g[k]$. Applying a similar procedure as for the OF method, considering the vector of $x[k]$ (represented as $\mathbf{x}$ ) time samples, the $j$ set of amplitudes $\hat{\mathbf{a}}_{j}$ can be given by

$$
\hat{\mathbf{a}}_{j}=\mathbf{U}_{j}^{T} \mathbf{x},
$$

where

$$
\mathbf{U}_{j}=\mathbf{C}_{j}^{-1} \mathbf{G}_{j}\left(\mathbf{G}_{j}^{T} \mathbf{C}_{j}^{-1} \mathbf{G}_{j}\right)^{-1} .
$$

The $\mathbf{G}_{j}$ parameter corresponds to the matrix of shifted versions of TileCal reference pulse, where $j$ is the number of collisions within the calorimeter readout window, and $\mathbf{C}_{j}$ is the noise covariance matrix. When $j=N$, the number of collisions is equal to the size of the readout window, thus the estimator will take the form

$$
\hat{\mathbf{a}}=\mathbf{G}_{j}^{-1} \mathbf{x} .
$$

It is also worth pointing out that this expression does not depend on the noise covariance matrix $\mathbf{C}$, which is one of the advantages of the COF method over the OF method. Finally, COF method applies a linear cut to select only the amplitudes above a predefined threshold, defined in the filter design. This step aims at re-designing COF through Eq. (10), avoiding estimating signals without information (noise), and improving the amplitude estimates with relevant information. The drawback is that signals outside the readout window are not considered in the COF design. 


\subsection{The Wiener Filtering}

A third recently implemented and validated approach for energy estimation in TileCal readout channels is based on the Wiener filtering. In this method, a digital linear filter $c[0], c[1], \ldots, c[N-1]$ is designed, where the output $y[N]$ provides an estimate of the desired response $d[n]$ (acquired through simulation), given an input signal with $N$ elements $x[0], x[1], \ldots, x[N-1]$. The Wiener filter design aims to minimize the mean square value of the estimation error, which leads to a mathematically more treatable problem. Unlike the OF method, the Wiener filter considers the uncertainties from the signal and the noise in its minimization process. In particular, this criterion based on the mean square error results in a second-order dependence of the cost function on the filter coefficients. Furthermore, the cost function has a distinct global minimum that defines singularly the optimal design of the filter, in the statistical sense [21]. In this approach, the filter output is given by the sum

$$
y[n]=\sum_{k=0}^{N-1} c[k] x[n-k],
$$

such that the error between the desired value and the estimated value $e[n]=$ $d[n]-y[n]$ is minimized. To optimize the filter design, the criterion of minimizing the mean square error was adopted. For this, the following cost function is defined by

$$
\mathbf{J}=\mathbb{E}\left\{e[n]^{2}\right\} .
$$

The minimum of the cost function $\mathbf{J}$ in respect to the coefficients $c[k]$ is given by

$$
\sum_{i=0}^{N-1} c[i] \mathbb{E}\{x[n-k] x[n-i]\}=\mathbb{E}\{x[n-k] d[n]\},
$$

where $k=0,1, \ldots, N-1$. From Eq. (14), it can be seen that:

1. The expected value $\mathbb{E}\{x[n-k] x[n-i]\}$ is the auto-correlation function of the filter input for the $i-k$ lag. This expression can be rewritten as

$$
R[i, k]=\frac{1}{N} \sum_{n=0}^{N-1} x[n-k] x[n-i] .
$$

2. The expected value $\mathbb{E}\{x[n-k] d[n]\}$ is the cross-correlation between the filter input and the desired output for the $i-k$ lag. This expression can also be rewritten as

$$
p[k]=\frac{1}{N} \sum_{n=0}^{N-1} x[n-k] d[n] .
$$

Equations (15) and (16) are known as the Wiener-Hopf equations. It should be stressed that for nongaussian noise (such as pile-up), this method operates in sub-optimal conditions. Substituting these equations in Eq. (14), a linear 
equation system is obtained as a necessary and sufficient condition to optimize the filter, as follows

$$
\sum_{i=0}^{N-1} c[i] R[i, k]=p[k] \quad k=0,1, \ldots, N-1 .
$$

Finally, this equation system can be rewritten in the matrix form and the Wiener filter optimal weights can be expressed by

$$
\mathbf{c}=\mathbf{R}^{-1} \mathbf{p},
$$

where $\mathbf{R}$ represents the auto-correlation matrix of the input signals samples (Eq. (15)) and $\mathbf{p}$ represents the cross-correlation matrix between the input signals samples and the desired values for the filter output (Eq. (16)).

It is worth mentioning that Wiener filter results in the optimal filter in the sense of minimizing the mean square error (error dispersion), taking into account the statistics present in the input data (signal plus noise). However, the Wiener filter considers that the noise has a zero mean (average value), which does not correspond to the case of uni-polar signals pile-up noise. To circumvent the noise average problem, an additional coefficient is included in the Wiener filter optimization process. This additional element of constant value equal to 1 is added to each input signal as the last element. In this way, the input signal has $N+1$ elements and the coefficient vector is also increased by one element.

The goal of including this additional element is to cancel the independent component of the signal in the optimization procedure, absorbing the average noise value in order to compensate for its contribution in the amplitude measurement of a given readout window. As a result, the estimation of the $\hat{A}_{F W}$ amplitude of the proposed Wiener filter is given by the sum of the products of the received signal temporal samples and the first $N$ coefficients of c. At the end of the operation, the last coefficient $c[N]$ is added to the result, compensating for the average noise value as shown in Eq. (19).

$$
\hat{A}_{F W}=\left(\sum_{i=0}^{N-1} c[i] x[i]\right)+c[N] .
$$

\section{Results}

Real proton-proton collision data acquired in 2018 by the LHC (last data acquisition period) were used for performance evaluation of the described methods [22]. Different levels were tested, with an average number of interactions per collision $\langle\mu\rangle \approx 30,\langle\mu\rangle \approx 40,\langle\mu\rangle \approx 50$, and $\langle\mu\rangle \approx 90$. The datasets for each case contain about 1 million events. For $\langle\mu\rangle \approx 30$ and $\langle\mu\rangle \approx 50$, the Wiener filter method was designed using a dataset of $\langle\mu\rangle \approx 40$, in order to profit of the number of signals used to design the methods. For $\langle\mu\rangle \approx 90$, the dataset was divided equally into two subsets, the development set (used to design the methods), and the test set (used to evaluate their efficiency). Since the OF and COF 
methods use only the pulse shape information in their models, the same design of each method was used for all $\langle\mu\rangle$ values.

It is worth mentioning that the data used were obtained from datasets called ZeroBias Stream, where only random triggers are used. Therefore, only electronic noise and pile-up information were acquired. In other words, in these events it is not expected to observe any signal of interest in the acquisition window, constituting only noise data. Hence, the mean value and RMS of the energy estimation of these events represent, respectively, the estimation bias and variance associated with each algorithm.

The Wiener filter is designed from a dataset composed of signals of interest immersed in noise, as well as the known amplitude values of the respective signals. Therefore, a pulse simulator was developed and validated to produce a dataset of signals of interest with the known amplitudes values, considering both pulse deformation and phase shift uncertainties. The amplitude follows a uniform distribution in the range of $[0,1023]$ ADC counts, since TileCal's analogto-digital converter has 10 bits [14]. Thus, each amplitude value has the same probability of occurrence. Finally, the generated signals were added to the events of the dataset used to derive the Wiener filter coefficients.

\subsection{Efficiency Analysis}

To analyse the efficiency of the studied filters for severe conditions, the most affected cells in terms of effect in TileCal, called E4 cells, were used. Figures (3a) and (3b) show the reconstructed energy distributions by the Wiener filter, COF, and OF2, considering $\langle\mu\rangle \approx 50$ and $\langle\mu\rangle \approx 90$, respectively. It can be seen the histograms for the Wiener filter shows less dispersion followed by the COF method. This result shows that for channels with high pile-up levels the Wiener filter presents a promising performance.

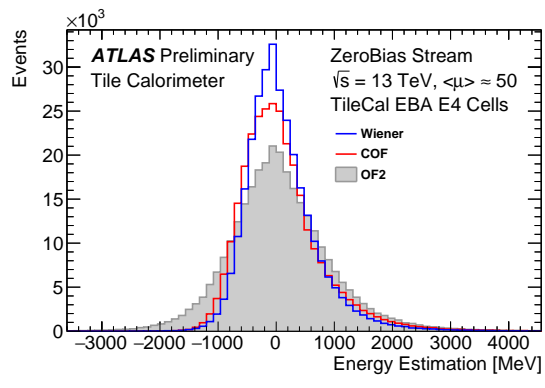

(a)

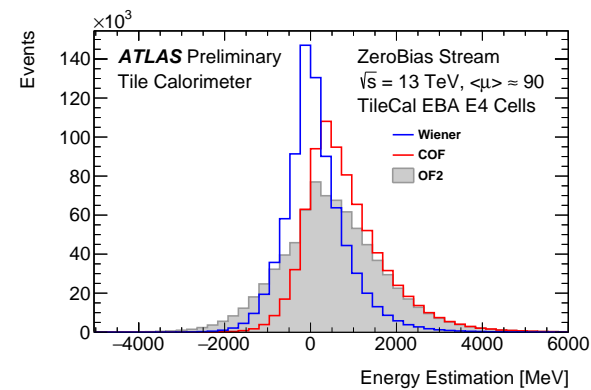

(b)

Fig. 3: Reconstructed energy distribution for $(\mathrm{a})\langle\mu\rangle \approx 50$ and $(\mathrm{b})\langle\mu\rangle \approx 90$ (extracted from [23]). 
The evolution of the mean and RMS of the estimation error distributions according to the $\langle\mu\rangle$ value are shown in Figures (4a) and (4b), respectively. Again, the Wiener filter has a lower mean and RMS compared to the COF and OF2 filters in the highest occupancy readout cells. For instance, for $\langle\mu\rangle \approx 90$, the Wiener filter shows an improvement of approximately $20 \%$ and $35 \%$ in terms of RMS compared to the COF and OF2 filters, respectively.

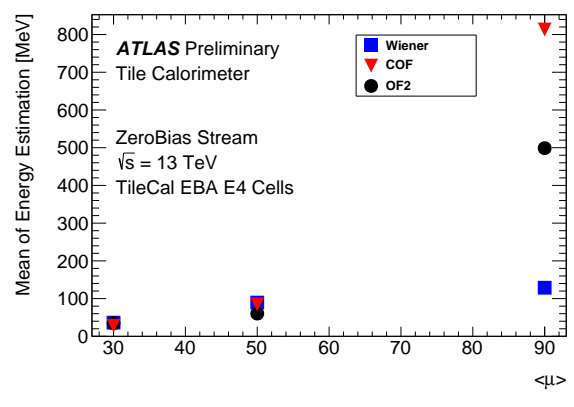

(a)

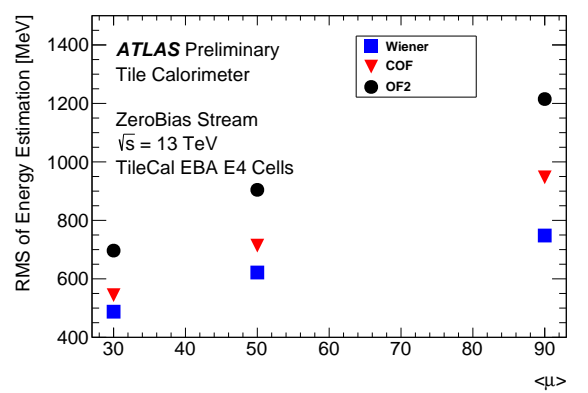

(b)

Fig. 4: Evolution of the (a) mean and the (b) standard deviation of reconstructed energy distribution with collision data (extracted from [23]).

In order to verify the estimation performance in other TileCal channels, a complete module was used (Module 01 of the EBA partition). In this analysis, the bias and variance of the three methods are compared. Fig. (5a) shows the difference in the estimated energy distribution mean between the Wiener filter and COF, using the OF2 method as a reference. Positive values represent higher mean values than OF2 and negative values represent lower values. For example, considering channel 1 (E4 cells), the reference method (OF2) presents a larger mean error with respect to the Wiener Filter (see Fig. (4a)), which produces the negative values for the data-points shown in Fig. (5a).

Fig. (5b) shows the relative percentage difference of the standard deviation of the energy distribution of the methods, adopting the OF2 as a reference and considering only one module. Once again, it is noted that the most significant improvements achieved by using the Wiener filter with respect to COF and OF2 are visible for channels 0 and 1 (cells of highest occupancy in the module). For the other channels, the COF method proved to be more efficient, surpassing the Wiener and OF2 filter, improving the RMS by approximately $25 \%$ with respect to the OF2 method. 


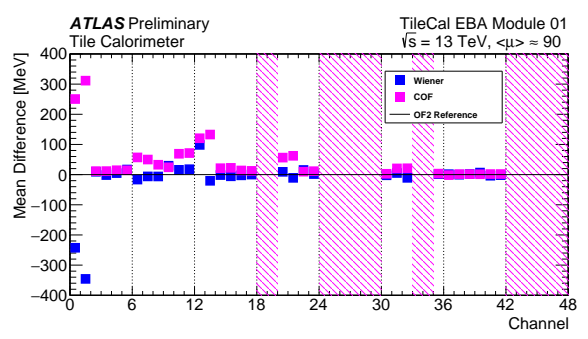

(a)

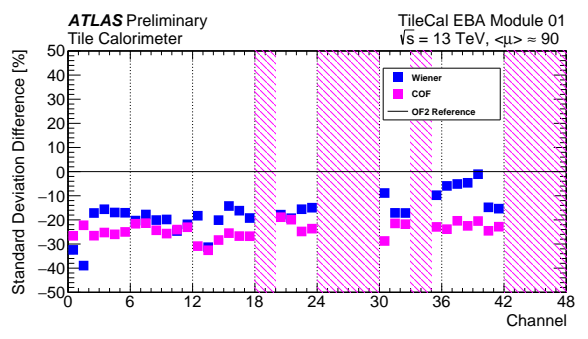

(b)

Fig. 5: Variation of the (a) difference of mean and (b) relative deviation from the standard deviation of the energy distribution for the Wiener and COF filters, adopting the OF2 method as a reference. Only Module 1 of TileCal's EBA partition is considered. Hatched areas correspond to non-instrumented channels (extracted from [23]).

\section{Conclusions}

This paper addressed the parameter estimation problem applied to energy reconstruction in the ATLAS Tile calorimeter cells under high pile-up conditions which introduce new challenges for the energy estimation task.

Using collision data for severe pile-up conditions $(\langle\mu\rangle \approx 90)$, the Wiener filter method shows an improvement of approximately $35 \%$ on the uncertainty of the energy reconstruction compared to the currently used method (OF2), in the most occupied TileCal cells. It was also observed that for low and medium occupation ranges, the COF method presents the best performance for energy estimation, improving the estimation variance by approximately $25 \%$ with respect to OF2.

The impact of the different energy reconstruction techniques on the physics objects reconstruction is under study.

\section{Acknowledgments}

The authors thanks to CNPq, RENAFAE (MCTIC), FAPERJ, FAPEMIG and FAPESB for the financial support. This study was financed in part by the Coordenação de Aperfeiçoamento de Pessoal de Nível Superior - Brasil (CAPES) Finance Code 001.

\section{References}

1. R. Wigmans, Calorimetry: Energy measurement in particle physics. Oxford University Press, 2017.

2. G. F. Knoll, Radiation Detection and Measurement. John Wiley \& Sons, 2010.

3. E. Fullana et al., "Digital signal reconstruction in the ATLAS hadronic tile calorimeter," IEEE transactions on nuclear science, vol. 53, no. 4, pp. 2139-2143, 2006. 
4. P. Adzic et al., "Reconstruction of the signal amplitude of the CMS electromagnetic calorimeter," Eur. Phys. J. C, vol. 46S1, pp. 23-35, 2006.

5. L. R. Evans and P. Bryant, "LHC Machine," JINST, vol. 3, p. S08001. 164 p, 2008.

6. W. Herr and B. Muratori, "Concept of luminosity," 2006.

7. Z. Marshall, "Simulation of pile-up in the ATLAS experiment," Journal of Physics: Conference Series, vol. 513, no. 2, p. 022024, Jun 2014.

8. L. M. Andrade, Filho, B. S. Peralva, J. M. de Seixas, and A. S. Cerqueira, "Calorimeter response deconvolution for energy estimation in high-luminosity conditions," IEEE Transactions on Nuclear Science, vol. 62, no. 6, pp. 3265-3273, 2015 .

9. D. P. Barbosa, L. M. d. A. Filho, B. S. Peralva, A. S. Cerqueira, and J. M. de Seixas, "Sparse representation for signal reconstruction in calorimeters operating in high luminosity," IEEE Transactions on Nuclear Science, vol. 64, no. 7, pp. 1942-1949, 2017.

10. D. Oliveira Goncalves, "Energy reconstruction of the ATLAS Tile Calorimeter under high pile-up conditions using the Wiener Filter," CERN, Geneva, Tech. Rep. ATL-TILECAL-PROC-2019-002, May 2019. [Online]. Available: https://cds.cern.ch/record/2674807/

11. ATLAS Collaboration, "The ATLAS Experiment at the CERN Large Hadron Collider," JINST, vol. 3, p. S08003. 437 p, 2008.

12. ATLAS Collaboration, "Readiness of the ATLAS Tile Calorimeter for LHC collisions," Eur. Phys. 346 J. C, vol. 70, pp. 1193-1236, 2010.

13. CERN, "Tile Calorimeter Public Plots," April 2013, last accessed on 26 May 2021. [Online]. Available: https://twiki.cern.ch/twiki/bin/view/AtlasPublic/ApprovedPlotsTile

14. K. Anderson et al., "Design of the front-end analog electronics for the ATLAS tile calorimeter," Nuclear Instruments and Methods in Physics Research Section A: Accelerators, Spectrometers, Detectors and Associated Equipment, vol. 551, no. 2-3, pp. 469-476, 2005.

15. F. Pastore, "The ATLAS Trigger System: Past, Present and Future," Nuclear and Particle Physics Proceedings, vol. 273-275, pp. 1065 - 1071, 2016, 37th International Conference on High Energy Physics (ICHEP).

16. B. Schmidt, "The high-luminosity upgrade of the LHC: Physics and technology challenges for the accelerator and the experiments," Journal of Physics: Conference Series, vol. 706, p. 022002, Apr 2016.

17. P. Klimek, "Quality Factors in TileCal and out-of-time Pile-up," Oct 2011. [Online]. Available: http://cds.cern.ch/record/1392389

18. D. Oliveira Damazio, "Signal Processing for the ATLAS Liquid Argon Calorimeter : studies and implementation," CERN, Geneva, Tech. Rep. ATL-LARG-PROC2013-015, Nov 2013. [Online]. Available: https://cds.cern.ch/record/1630826

19. M. Delmastro, "A stand-alone signal reconstruction and calibration algorithm for the ATLAS electromagnetic calorimeter," in 2003 IEEE Nuclear Science Symposium. Conference Record (IEEE Cat. No. 03CH37515), vol. 2. IEEE, 2003, pp. 1110-1114.

20. A. V. Oppenheim, Discrete-time signal processing. Pearson Education India, 1999.

21. S. O. Haykin, Adaptive filter theory. Pearson, 2013.

22. A. R. Martínez, "The run-2 ATLAS trigger system," Journal of Physics: Conference Series, vol. 762, p. 012003, Oct 2016.

23. CERN, "Tile Calorimeter Public Plots for Collision Data," January 2020, last accessed on 26 May 2021. [Online]. Available: https://twiki.cern.ch/twiki/bin/view/AtlasPublic/TileCaloPublicResults 